\title{
Reappraisal of the tribosphenidan mammals from the Trinity Group (Aptian-Albian) of Texas and Oklahoma
}

\author{
Brian M. Davis and Richard L. Cifelli \\ Acta Palaeontologica Polonica 56 (3), 2011: 441-462 doi: http://dx.doi.org/10.4202/app.2011.0037
}

The Trinity therians have long been the focus of attempts to reconstruct the evolutionary history of higher mammals, especially in the context of the development of tribospheny. In this paper, we update the taxonomy of the tribosphenidan taxa known from the Trinity Group and establish with more confidence the premolar/molar count in each. Many isolated specimens can be referred to a specific tooth locus. Additional diversity is revealed within the Deltatheroida, with the description of an additional species of Oklatheridium ; Pappotherium is here considered a likely metatherian based on the inferred presence of four molars, while Holoclemensia is a basal eutherian (the opposite of some traditional interpretations). The remainder of the genera, Kermackia and Slaughteria, cannot be allied with either of the living groups of tribosphenidan mammals using the available data. We identify strong morphological diversity within this assemblage of stem taxa, including modifications to the traditional tribosphenic occlusal pattern in Kermackia. Mammalian evolution at the base of the tribosphenidan radiation was complex, and this underscores the need for caution when interpreting the morphology and relationships of taxa known by incomplete material.

Key words: Tribosphenida, Metatheria, Eutheria, Deltatheroida, Trinity Group, Early Cretaceous.

Brian M. Davis [bmdavi@ou.edu] and Richard L. Cifelli [rlc@oul.edu], Department of Zoology and Sam Noble Oklahoma Museum of Natural History, University of Oklahoma, 2401 Chautauqua Ave, Norman, OK, 73072, USA.

This is an open-access article distributed under the terms of the Creative Commons Attribution License (for details please see creativecommons.org), which permits unrestricted use, distribution, and reproduction in any medium, provided the original author and source are credited. 
Fof Full text $(1,351.2 \mathrm{kB})$ 